\title{
«Migros-Wald» oder Märchenwald? (Essay)
}

Oliver Thees Eidgenössische Forschungsanstalt für Wald, Schnee und Landschaft (CH)*

\author{
«Migros-Wald» oder Märchenwald? (Essay)
}

Für die Schweizer Forstbetriebe wird es immer schwieriger, die an den Wald gestellten Anforderungen wirtschaftlich zu erfüllen. Diese Situation wird produktions-, industrie- und institutionenökonomisch analysiert, um der Vielschichtigkeit der Problematik gerecht zu werden. Die Schweizer Forstbetriebe sind Mehrproduktunternehmen in öffentlicher Hand. Deren Ziel ist es, für die Volkswirtschaft wichtige Ökosystemdienstleistungen in Form privater und öffentlicher Güter zu erzeugen. Dies geschieht eng verknüpft mit der Holzproduktion. Die Effizienz der Bereitstellung der Güter lässt sich noch verbessern, vor allem durch organisatorische Massnahmen, die durch Kooperation und Informationstechnologien ermöglicht werden. Schwieriger, aber unabdingbar, ist die vollständige Vergütung der bestellten öffentlichen Güter. Hierzu fehlen Anreize, marktähnliche Strukturen und Instrumente. Der Beitrag ist ein Plädoyer, öffentliche Güter unter privatwirtschaftlichen Bedingungen zu erzeugen, Strukturen der Bewirtschaftung zu verändern, Ansprüche auch zu senken und marktähnliche Abgeltungsmechanismen für die öffentlichen Güter zu entwickeln.

Keywords: public goods, payment for ecosystem services, multi-product firms, forestry, forest management doi: 10.3188/szf.2016.0200

* Zürcherstrasse 111, CH-8903 Birmensdorf, E-Mail oliver.thees@wsl.ch

$\mathrm{D}$ ie Schweiz gilt seit Jahren als eines der wettbewerbsfähigsten Länder der Welt. Der Schweizer Wald dagegen erhält zwar gute Noten für seinen ökologischen Zustand und für seinen grossen gesellschaftlichen Nutzen, nicht aber für die ihn bewirtschaftenden Forstbetriebe und ihre Holzproduktion: Sie gelten als wenig oder nicht wettbewerbsfähig. Um der Globalisierung und ihren ökonomischen Herausforderungen zu begegnen, wird aber genau das verlangt. Es stellt sich die Frage, wieso die Forstbranche unter den gleichen Rahmenbedingungen nicht auch wettbewerbsfähig sein kann. Wesentlich hierfür ist einerseits die Effizienz der Leistungserstellung und andererseits, dass die erbrachten Leistungen auch vollumfänglich abgegolten werden. Auf der Suche nach Antworten geht es also um grundsätzliche Fragen wie, was für wen produziert werden soll, wer diese Leistungen wie bezahlt, und wie sie mit welcher Art Wald erzeugt werden sollen - mit einem an Bedarfen, Knappheiten und wirtschaftlichen Erfordernissen orientierten «MigrosWald» oder mit einem an Wünschen orientierten Märchenwald.

\section{Forstbetriebe als Mehrprodukt- unternehmen}

Forstbetriebe produzieren private Güter wie Holz und öffentliche Güter wie Schutz und Erholung. Durch Produktion und Verkauf privater Güter erzielen die Forstbetriebe Erlöse. Holz, das wichtigste dieser Güter, wird auf internationalen und regionalen Märkten gehandelt. Hier bildet sich dessen Preis. Für öffentliche Güter gibt es dagegen keine Märkte und folglich auch keine Marktpreise. Ihre Bereitstellung wird zum Teil durch Abgeltungen und Subventionen durch den Staat vergütet und durch die Holzerlöse (quer)finanziert. Bereitgestellt werden sämtliche Güter des Waldes in der Schweiz durch öffentliche, vor allem kommunale Betriebe und durch private Waldeigentümer. Der volkswirtschaftliche Wert der öffentlichen Güter ist gross und übersteigt den der privaten. Die öffentlichen Güter werden immer bedeutsamer; ein Beispiel eines solchen Gutes ist der Klimaschutz. Hier wirkt der Wald durch sein Wachstum wie auch durch seine Nutzung positiv: Beide dämpfen den Klimawandel und seine Folgen. 


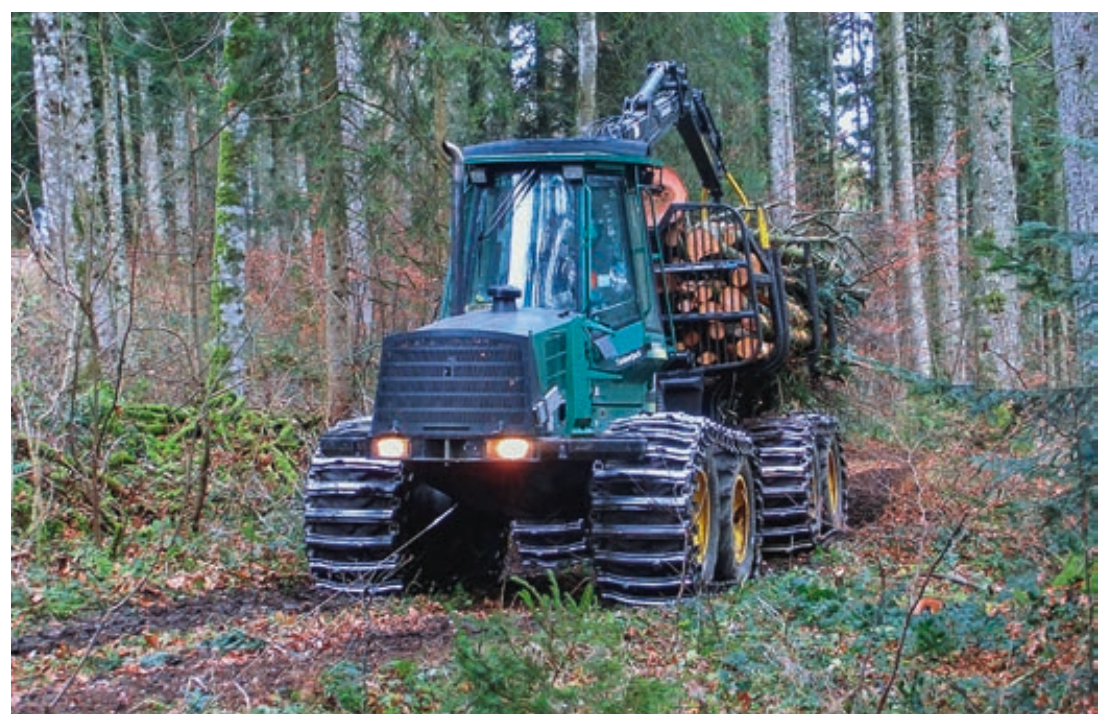

Abb 1 Die Erzeugung öffentlicher Güter wie Schutz vor Naturgefahren, Erholung, Klimaschutz und Biodiversität ist häufig mit der Holzproduktion eng verzahnt. Foto: Fritz Frutig, wSL

Forstbetriebe sind also Mehrproduktunternehmungen. Sie zeichnen sich durch eine besondere Heterogenität der Produkte aus: Sie produzieren private und öffentliche Güter und sind dabei sowohl in der Urproduktion als auch im Dienstleistungssektor tätig. Und sie tun dies gleichzeitig und gleichörtlich. Beide Güterarten werden in langen Zeiträumen erzeugt. Die Produktionsziele sind zum Teil konfliktär, sodass für deren Erreichung Optimierungen gefunden und Kompromisse geschlossen werden müssen. Offenbar bestehen aber auch beträchtliche Verbundeffekte (economies of scope), die dafür sprechen und es ermöglichen, diese doch sehr unterschiedlichen Güter integral effizient zu erzeugen. Erklären lassen sich diese durch Transaktionskosten, welche bei getrennter Produktion entstünden.

Die Produktion des privaten Gutes Holz und die Produktion der öffentlichen Güter sind sehr eng miteinander verbunden, sogar regelrecht verzahnt. Das verbindende Glied ist die Holzproduktion (Abbildung 1): Während Biodiversität und Erholung vor allem über monetäre Holznutzungsverzichte erreicht werden, geht es beim Schutz vor Naturgefahren vor allem um die Steuerung der Waldstrukturen, wofür Bäume gefällt und gegebenenfalls entnommen werden. Bezüglich Klimaschutz verspricht die stoffliche und energetische Nutzung des Holzes für die $\mathrm{CO}_{2}$ Speicherleistung innerhalb und ausserhalb des Waldes sowie für die Substitution fossiler Ressourcen langfristig ein besseres Ergebnis als der Nutzungsverzicht. Das Beispiel Klimaschutz und Energiewende zeigt, dass offenbar grosse Verbundeffekte bei der Produktion von privaten und öffentlichen Gütern vorhanden sind. Das bedeutet aber auch, dass Holzproduktion zunehmend von öffentlichem Interesse ist, und in diesem Fall die beiden Güterarten verschmelzen.

Man kann die privaten und öffentlichen Güter unter dem Begriff Ecosystem Services (Ökosys- temdienstleistungen) zusammenfassen und die Forstbetriebe als Organisationen auffassen, die diese durch Gestaltung und Lenkung des Ökosystems Wald erzeugen. Sie lassen sich in vier Kategorien einteilen (Millennium Ecosystem Assessment 2005): 1) unterstützende, ökosystemare Dienstleistungen wie der Nährstoffkreislauf, 2) bereitstellende Dienstleistungen wie Wasser und Holz, 3) regulierende wie Klima und 4) kulturelle wie Erholung und Landschaftsbild. Folgt man diesem Ansatz, gewinnt die Produktion der Forstbetriebe den Charakter umfassender Infrastrukturleistungen.

\section{Kostensituation: produktions- ökonomische Aspekte}

Verschiedene Faktoren verteuern bekanntlich die Schweizer Waldbewirtschaftung: die oft schwierigen Geländeverhältnisse, der hohe Preis des Produktionsfaktors Arbeit, die Kleinteiligkeit der Bewirtschaftung in der gesamten Produktionskette, der hohe Fixkostenanteil bedingt durch den hohen Anteil an Eigenregie, die hohen Ansprüche der Gesellschaft an die Waldbewirtschaftung etc. Letztere manifestieren sich in zahlreichen formellen und informellen Regeln, welche die Bewirtschaftung einschränken, Mehraufwand verursachen und die Produktion verteuern.

Die Holzproduktion ist aktuell defizitär, nicht nur im Gebirge, sondern vielfach auch im Mittelland bei besten Wachstumsbedingungen, günstigen Gelände- und Erschliessungsverhältnissen, wo man eigentlich tiefe Erntekosten und Gewinne im Holzproduktionsbetrieb erwartet. Gleichwohl ist es auch hier oft nicht möglich, die Erntekosten zu decken, geschweige denn mit der Holzproduktion die Erstellung der öffentlichen Güter zu finanzieren. Obwohl die Betriebe in der Vergangenheit rationalisiert haben, sind die Ergebnisse negativ. Es gibt jedoch noch Spielräume für weitere Verbesserungen. Wie in anderen Branchen der Urproduktion steht auch in der Holzproduktion die Kostensenkung im Vordergrund, wenn es um die Wettbewerbsfähigkeit geht. Besonders relevant hierfür ist die Holzernte. Hier fallen die meisten Kosten an; umliegende Länder produzieren günstiger.

Die öffentlichen Güter werden im Rahmen der Gestaltung und Lenkung des Ökosystems erzeugt. Es werden kostenwirksame Massnahmen ergriffen, um einen angestrebten Waldzustand zu erreichen. In der Regel handelt es sich um Mehraufwand gegenüber einer reinen Holzproduktion, hervorgerufen entweder durch Massnahmen, die der Erfüllung von ökologischen Mindestanforderungen bei der Holzproduktion dienen, zum Beispiel beim Bodenschutz, oder durch Massnahmen, die einer anderen Hauptfunktion des Waldes, etwa dem Schutz vor Naturgefahren oder des Trinkwassers, zugutekommen. Mitunter werden die Anforderungen sogar übererfüllt. 


\section{Erlössituation: industrieökonomische Aspekte}

Die Wald- und Holzwirtschaft führt wie andere Branchen in der Schweiz kein Inseldasein (mehr). Sie wird beeinflusst von den internationalen politischen und wirtschaftlichen Entwicklungen.

Das private Gut Holz unterliegt dem globalen Wettbewerb auf den Rohstoffmärkten. Das vergangene Jahrzehnt war von einem sogenannten Rohstoffsuperzyklus geprägt (Rohner 2016). ${ }^{1}$ Die Preise für Rohstoffe, allen voran Energieträger und Metalle, waren rasant gestiegen und verblieben mit einem Unterbruch während der Finanzkrise auf hohem Niveau. Diese Entwicklung scheint nun zu Ende zu gehen. Die Rohstoffpreise sinken auf das vorherige niedrige Niveau. Davon sind auch die Holzpreise betroffen. Sie ergeben sich aufgrund des Angebotes und der Nachfrage auf den internationalen Rohstoffmärkten. Dabei bestimmen weltweit der Industrieholz- und der Energieholzmarkt das Holzpreisniveau. Nur in Mitteleuropa dominiert der Sägeholzmarkt. Die internationalen Märkte bestimmen weitgehend auch das Holzpreisniveau im Inland. In der Schweiz haben wir es mit einem Nachfrageoligopol zu tun: Wenige grosse Käufer von Holz, vor allem Sägewerke, stehen einer Vielzahl von kleinen Verkäufern gegenüber. Entsprechend dieser schwachen Marktposition verhalten sich viele Forstbetriebe als Mengenanpasser. Die Holzpreise kommen durch diese Marktsituation im Inland eher noch weiter unter Druck. Die aktuelle Situation ist zudem gekennzeichnet durch die Frankenstärke, welche im Inland den Rundholzpreis drückt und Holzprodukte aus dem Ausland verbilligt. Der Export von Rund- und Schnittholz stagniert. Für die Wald- und Holzbranche ist es schwieriger geworden, international wettbewerbsfähig zu sein.

Die öffentlichen Güter werden im Rahmen der Gestaltung und Lenkung des Ökosystems erzeugt. Hierzu werden erlöswirksame Massnahmen ergriffen, um einen angestrebten Waldzustand zu erreichen. Oft gehen diese einher mit Holznutzungsverzichten, die zu Mindererlösen gegenüber der reinen Holzproduktion führen. Viele der öffentlichen Güter werden gesetzlich eingefordert. Eine Abgeltung der Erbringung erfolgt nicht in jedem Fall und ist, wenn sie erfolgt, am betrieblichen Aufwand und nicht am Wert des Gutes für den Abnehmer orientiert.

\footnotetext{
Abb 2 Ansätze zur Rationalisierung auf verschiedenen Handlungsebenen im Mehrproduktunternehmen Forstbetrieb (PES: Payment for Ecosystem Services).
}

\begin{tabular}{|l|c|c|}
\hline $\begin{array}{l}\text { Institutionelle Ebene } \\
\rightarrow \text { Anreize }\end{array}$ & Kooperation & PES \\
\hline $\begin{array}{l}\text { Organisatorische Ebene } \\
\rightarrow \text { Wettbewerbsposition }\end{array}$ & $\begin{array}{c}\text { Skalen- } \\
\text { effekte }\end{array}$ & $\begin{array}{c}\text { Verbund- } \\
\text { effekte }\end{array}$ \\
\hline $\begin{array}{l}\text { Ebene des Ressourcen- } \\
\text { einsatzes } \rightarrow \text { Kostensenkung }\end{array}$ & $\begin{array}{c}\text { Effizienz- } \\
\text { effekte }\end{array}$ & $\begin{array}{c}\text { Verbund- } \\
\text { effekte }\end{array}$ \\
\hline
\end{tabular}

\section{Situation des wirtschaftlichen Handelns: institutionenökonomische Aspekte}

Die institutionellen Rahmenbedingungen beeinflussen das wirtschaftliche Verhalten der Akteure. Sie ergeben sich durch 1) die Eigentumsverhältnisse und 2) die Regelungen zur Abgeltung von Leistungen im öffentlichen Interesse.

Der öffentliche Wald hat in der Schweiz einen vergleichsweise sehr hohen Anteil von rund 70\% und wird in der Hauptsache von kommunalen Forstbetrieben bewirtschaftet. Diese sind nicht erwerbswirtschaftlich ausgerichtet; Gewinnmaximierung ist nicht ihr Ziel. Dies gilt weniger für die Ortsbürgergemeinden (weil ohne Steuereinnahmen und daher bestrebt, keine roten Zahlen zu schreiben) und mehr für die politischen Gemeinden (weil mit Steuerhoheit). Ihre Zielsetzung stellt vielmehr darauf ab, für die Gesellschaft eine Vielzahl von Leistungen (inklusive Holzerzeugung) zu erbringen, wobei die Ansprüche an diese zunehmen. Viele der öffentlichen Güter werden wie erwähnt nicht abgegolten, und es gibt wenig Anreize, welche ihre Erbringung und die Effizienz der Erstellung finanziell attraktiv machen würden.

\section{Massnahmen zur Verbesserung der Wettbewerbsfähigkeit}

\section{Ansätze}

Die Wettbewerbsstrategien Kostenführerschaft, Differenzierung und Fokussierung nach Porter (1990) sind wie folgt einzuschätzen: In der Forstbranche dürfte die Kostenführerschaft (kostengünstigster Anbieter) am ehesten infrage kommen. Differenzierung (sich von Konkurrenten abheben) und Fokussierung (Nischenprodukte) sind vorstellbar, aber wahrscheinlich keine Rezepte für eine breite Umsetzung. Die Strategien basieren darauf, dass es Wettbewerb gibt. Dies trifft aber bei den öffentlichen Gütern definitionsgemäss nicht zu und bei den privaten nur teilweise. Trotzdem ist es sinnvoll, diese Ansätze zu prüfen und dabei den Verbund der Güterarten zu berücksichtigen.

Die konkreten Massnahmen lassen sich getrennt nach den Güterarten verschiedenen Handlungsebenen zuordnen (Abbildung 2):

\section{Die Massnahmen bei der Holzproduktion sind eher kurz- und mittelfristiger Art:}

Kosten lassen sich senken

- auf der Ebene des Ressourceneinsatzes durch Optimierung der biologischen Produktion (Extensivierung, starke Bejagung) und der Holzernte (Me-
1 Das Ende des Rohstoffsuperzklus. Finanz und Wirtschaft vom 15.1.2016. www.fuw.ch/article/das-ende-des-rohstoff-superzyklus (31.5.2016) 
chanisierung, Blockbildung, Innovationen im steilen Gelände) im Verbund mit der Erschliessung (Ausbaustandard 5-Achs-Lkw),

- auf der organisatorischen und institutionellen Ebene durch Nutzen von Grössenvorteilen (Economy of Scale) bei der Planung und Steuerung der gesamten Bewirtschaftung durch überbetriebliche Zusammenarbeit (Funktionalisierung), aber auch durch Verpachtung oder aussetzenden Betrieb.

\section{Erlöse lassen sich steigern}

- auf der Ebene des Ressourceneinsatzes durch ein optimiertes Baumartenportfolio (Douglasienanbau),

- auf der organisatorischen und institutionellen Ebene durch Nutzen von Grössenvorteilen (Economy of Scale) bei der Holzvermarktung (Wettbewerbsposition) und bei der Kundenorientierung durch überbetriebliche Zusammenarbeit.

\section{Die Massnahmen bei den öffentlichen Gütern sind} eher mittel- und langfristiger Art:

\section{Kosten lassen sich senken}

- auf der Ebene des Ressourceneinsatzes und der organisatorischen Ebene durch Optimierungen bei konfliktären Zielen unter Berücksichtigung ökonomischer Aspekte und durch betriebsübergreifende Planung, Steuerung und Durchführung der Massnahmen, wenn möglich im Verbund mit der Rationalisierung der Holzproduktion,

- auf der institutionellen Ebene durch Reduzieren der Ansprüche vornehmlich im Wirtschaftswald.

Erlöse lassen sich steigern

- auf der institutionellen Ebene durch eine vermehrte Abgeltung der Leistungen und gegebenenfalls durch Schaffung von künstlichen Märkten/Ersatzmärkten.

Schlüssel bzw. Enabler für die beiden Massnahmenpakete sind 1) die Kooperation der forstlichen Akteure, 2) die Informationstechnologie, 3) die Nutzung von Verbundeffekten zwischen den privaten und den öffentlichen Gütern und 4) die Schaffung von Anreizen für wirtschaftliches Handeln.

\section{Wertungen}

Die Forstwirtschaft ist integraler Bestandteil unserer Volkswirtschaft. Sie unterliegt der auf Wettbewerb aufgebauten Wirtschaftsordnung und kann sich deren Spielregeln nicht entziehen. Dies trotz einiger Besonderheiten, welche sie vom Rest der Wirtschaft unterscheidet. Daher kommt der Forstbetrieb nicht umhin, weiter zu rationalisieren bzw. sich an die wirtschaftlichen Erfordernisse anzupassen, wenn er überleben will. Auch die Tatsache, dass die Holznutzung immer mehr auch von öffentlichem Interesse ist, spricht dafür. Da man im internationa- len Vergleich manche Rationalisierungsschritte nicht gemacht hat, kann man von den Erfahrungen Dritter lernen und Fehler vermeiden.

Massnahmen auf Ebene Ressourceneinsatzes: Rationalisierungen in Form des Einsatzes leistungsfähiger Erntemaschinen und durch die optimale Gestaltung von Holzernte und Erschliessung bergen noch Potenziale für Kostensenkungen. Allerdings muss man sich bewusst sein, dass diese begrenzt sind. Mittel- und langfristig nähern sie sich einem Wert, der nicht weiter verbessert werden kann. Sie bedingen Investitionen und erhebliche Anpassungen in vielen betrieblichen Bereichen, vor allem auch im organisatorischen. Sie können mit Risiken für andere Güter des Waldes und für bestimmte Aspekte der Qualität verbunden sein. Sie können aber auch der Erzeugung öffentlicher Güter zugute kommen.

Massnahmen auf organisatorischer Ebene: Hier bestehen grössere Kostensenkungspotenziale. Ein Schlüssel liegt in der Zusammenarbeit der Betriebe, um Economies of Scale (Grössenkostenersparnisse) zu nutzen und so Nachteile der Kleinteiligkeit der Forststrukturen zu überwinden. Holzernte und -vermarktung bieten sich hierfür an und können zum Beispiel im Rahmen einer Funktionalisierung zentral durch eine Stelle für einen ganzen Kanton oder eine ganze Region wahrgenommen werden. Die modernen Informationstechnologien machen diese Lösungen überhaupt erst möglich und sind ein Schritt zur weiteren Professionalisierung. Diese Zusammenarbeiten bedingen aber die Aufgabe von Eigenständigkeit bzw. Kompetenzen und letztlich auch die Einsparung von Personal. Die erheblichen Spielräume, um Skaleneffekte zu erzielen, sollten genutzt werden, sich aber nicht allein auf die Betriebs- bzw. Reviergrösse beschränken und diese zu maximieren suchen. Sie sollten vielmehr fokussieren auf grössere, gut kooperierende Einheiten.

Massnahmen auf institutioneller Ebene: Grundlegend für die Verbesserung der Wettbewerbsfähigkeit ist erstens, dass ordnungspolitisch die Möglichkeit zur Anpassung gewährleistet wird, dass den Bewirtschaftern ein möglichst grosser Spielraum an Gestaltungsmöglichkeiten des Betriebes und der Vermarktung offensteht. Hierzu ist es wichtig, dass dieser nicht durch zusätzliche, über das Gesetz hinausgehende Vorgaben eingeengt wird und dass die Ansprüche der Öffentlichkeit vor allem im Wirtschaftswald reduziert werden. Behörden und Zertifizierer sollten hier im Sinn einer umfassenden Nachhaltigkeit handeln und den Ausgleich suchen. Viele der Ziele sind konfliktär, und es müssen Kompromisse gefunden werden. Zweitens sind die bestellten öffentlichen Güter vollumfänglich abzugelten (Payment for Ecosystem Services); für deren Sicherung sind auch Überschüsse notwendig (zum Beispiel Forstreserve). Hierzu sind Anreize zu schaffen, zum Beispiel durch künstliche Märkte (institutionelles 
Unternehmertum; Schlüter \& Nichiforel 2010), die wirtschaftliches Handeln erfordern und belohnen. Die Entwicklung von Indikatoren und Bewertungen für öffentliche Güter sind ein erster Schritt. Da diese im Verbund mit dem privaten Gut Holz erzeugt werden, könnte hierin nicht nur ein Problem, sondern auch ein Schlüssel für innovative Lösungen liegen.

Zur Umsetzung dieser Ansätze sind Änderungen der privat- und öffentlich-rechtlichen Rahmenbedingungen erforderlich (Zimmermann 2010).

\section{Folgerungen}

Um die Wettbewerbsfähigkeit der Schweizer Forstbetriebe und damit ihren Beitrag für die Volkswirtschaft zu verbessern, ist an mehreren Stellschrauben zu drehen:

- Kosten senken in der Urproduktion, vor allem durch Kooperation und IT-Einsatz in der Produktionskette sowie durch Reduktion der Ansprüche der Öffentlichkeit im Wirtschaftswald.

- Erlöse verbessern im Dienstleistungsbereich, durch vollständige Bezahlung der öffentlichen Güter im Rahmen neuer Anreizsysteme, zum Beispiel durch künstliche Märkte.

- Frei(er)es Bewirtschaften ermöglichen, um optimal angepasste Lösungen zu erzielen: Deren Spanne reicht vom aussetzenden Betrieb über suffizienzorientierte Bewirtschaftung bis zur intensiven Holzproduktion.

- Umdenken: den Forstbetrieb als Mehrproduktunternehmung, als Provider von Ecosystem Services verstehen und dabei die ausgeprägten Verbundeffekte nutzen.

Die Migros als Vergleich mag der Försterei aufs Erste nicht gefallen. Sie steht aber für ein erfolgreiches, modernes Konzept, in dem Economies of Scale in der Produktion und Economies of Scope in der Vermarktung die Strategie bestimmen. Migros steht für einen Weg, Leistungen kostengünstig und zugleichinguterQualitätanzubieten.Im Märchen(wald) gehen zwar alle Wünsche in Erfüllung; aber das ist nicht die Realität, nur manchmal ist es wie im Märchen, d.h. bezahlbar. Während Skandinavien das Mekka der Holzproduktion darstellt, sollte die Schweiz eine Pilgerstätte werden, wo man sehen kann, wie man mit den hohen Anforderungen der postindustriellen Gesellschaft an die Forstwirtschaft mustergültig, d.h. nachhaltig und damit auch wirtschaftlich, zurechtkommt.

Eingereicht: 17. Mai 2016, akzeptiert (ohne Review): 31. Mai 2016

\section{Literatur}

MILLENNIUM ECOSYSTEM ASSESSMENT (2005) Ecosystems and human well-being: a framework for assessment. Washington DC: Island Press. 245p.

PORTER ME (1990) Wettbewerbsstrategie, Methoden zur Analyse von Branchen und Konkurrenten. Frankfurt a.M.: Campus, 6 ed. 488 p.

SCHLÜTER A, NICHIFOREL L (2010) Gemachte Märkte: institutionelles Unternehmertum im Forstsektor. Schweiz Z Forstwes 161: 355-361. doi: 10.3188/szf.2010.0355

ZIMMERMANN W (2010) Rechtliche Aspekte bei der Vermarktung von Nichtholz-Waldleistungen. Schweiz Z Forstwes 161: 362-367. doi: 10.3188/szf.2010.0362

\section{Fairytale forests must face reality (essay)}

Swiss forest enterprises are finding it increasingly difficult to fulfill the demands on the forest economically. The problem is complex. To address it, we analyzed this situation from the points of view of production, industrial and new institutional economics. Swiss forest enterprises are multi-product firms. They are usually publicly owned and aim to provide crucial ecosystem services for the economy in the form of private and public goods that are mostly closely connected with the production of wood. Providing these goods can be made more efficient, especially by adopting organizational measures involving cooperation and information technologies. Another more difficult but necessary measure is to ensure the required public goods are paid for. No incentives, market-like structures or tools for this have yet been introduced. This paper is a plea for providing public goods under private-sector conditions, changing management structures accordingly, even reducing the demands on the forest and developing market-based mechanisms for paying for the public goods. 